\title{
3D IMAGES FOR AUTOMATED DIGITAL ODONTOMETRY
}

\author{
A.V. Gaboutchian ${ }^{1}$, V. A. Knyaz ${ }^{2,3}$ \\ ${ }^{1}$ Moscow State Medical-Stomatological University, Moscow, Russia - armengaboutchian@ mail.ru \\ 2 State Research Institute of Aviation System (GosNIIAS), 125319 Moscow, Russia - knyaz@gosniias.ru \\ ${ }^{3}$ Moscow Institute of Physics and Technology (MIPT), Russia
}

Technical Commission II

KEY WORDS: Automated digital odontometry, Photogrammetry, Intraoral scanner, Cone Beam Computed Tomography, X-ray micro computed tomography, Odontometrics, Anthropology, Palaeoanthropology

\begin{abstract}
:
Improvements of existing and development of new non-contact measurement techniques, especially for surfaces of complex spatial shape, allow involvement of various disciplines into advanced technological reality. These improvements have two major directions. The first, being more obvious, refers to introduction of accurate digital 3D images in spheres where real objects have become subjects of traditional study, techniques or manufacturing technologies. The other direction deals with substantial methodological improvements, as they become possible only with introduction of the above-mentioned techniques. Among such is the division of physical anthropology, of dentistry and other disciplines related to dental studies, - odontometry, or measurements of teeth. Traditional odontometry, by turning into automated digital odontometry, becomes a method of accurate and objective morphological assessments in dentistry and anthropology, including palaeoanthropology. As a new method, automated digital odontometry requires interpretations of dental morphology (applicable in digital techniques), accurate 3D images of teeth and software based on 3D and 2D image analysis suitable for automated measurements. The mentioned factors are particularly important for this method due to its inapplicability on real objects. Thus various approaches to obtaining digital images are discussed in the context of their quality and conformity with the studied material and odontometric technique, which currently includes automated orientation algorithms setting locations for principal morphological structures and measurement algorithms themselves, likewise functioning in an automated mode.
\end{abstract}

\section{INTRODUCTION}

Digital imaging of teeth and surrounding tissues is a matter of wide and versatile discussions in scientific literature. Being at high stage of its fast development, it is in active progress, process of revisions regarding applications, software, precision, image quality and other factors due to various issues, such as involving new fields of implementation and necessity of permanent technological improvements (e.g., dose reduction in computed tomography). Implementation of 3D images in certain cases is in itself progress, providing previously unavailable data (as, for instance, visualisation of concealed morphological features). They can also provide for effective substitution of existing techniques or improvements in existing techniques (such as arch measurements in orthodontics). In other cases they facilitate development of new techniques, like in odontometric studies.

For many years past, odontometry has been the method of expertbased estimation of tooth dimensions though application of calipers and their correct, in accordance to recommendations developed in the pre-digital era, positioning on surfaces of measured objects. Being applied in physical anthropology or dentistry to this day (Shaweesh, 2016), (Shireen and Ara, 2016), (Song et al., 2017), methodological fundamentals of traditional have been developed in late nineteenth - middle twentieth centuries by a number of prominent anthropologists (Zubov, 1968), (Irish and Scott, 2015) have had contributed significantly to the method as well as. The most obvious approach is in application of existing measurements

\footnotetext{
${ }^{*}$ Corresponding author
}

techniques on digital images. It encourages by its results, including improvements in odontometry (enabling contour measurements, for instance) (Smith et al., 2009), (Naidu and Freer, 2013a), (Naidu and Freer, 2013b) on the other hand such studies are necessary for introductions of new techniques or devices (Rajshekar et al., 2017).

At the same time positive features of digital workflow facilitate odontometric process (data storage and sharing, communication, risks of wear, loss or breakage minimisation etc.). However, such approaches, as extensions of traditional methods, keep not only their fundamental principles but limitations as well and thus, referring to odontometry, they do not provide enough data (neither in terms of quantity, nor quality) to have a closer view on morphological features of teeth. This task traditionally has been solved in odontology through descriptions of morphological features based on visual examination of teeth, which has always been characterised by a certain degree of subjectivity, especially in "level of feature expression" terms. Thus availability of method combining the objectivity of measurements with possibility of morphological description is important in odontology, which coincides with vector of automated digital odontometry development. It would be misleading to compare directly odontoscopic (visual, or non-metric) studies with automated digital odontometry. As they are differ in basic approaches there are parametrs and features that cannot be related, at least at the moment, to both methods. Nevertheless, automated digital odontometry provides data on morphology, which has been described visually remaining unattainable for manual measurements (Gaboutchian et al., 2019). At the same time automated digital odontometry gener- 
ates datasets, referring even to a single tooth or its morphological structures, orders greater by volume than traditional odontometry does. Such datasets are used for characterising individual teeth as well as their typically arranges groups (molars or premolars, upper or lower teeth) morphological features, as well as reflect morphological changes caused by process of attrition (within one individual, when data is collected with significant time intervals, or between groups of teeth or individuals). Variety of parameters includes linear, angular, contour, area measurements and calculations of various coefficients; and this is only a part of the method potential, as odontometric part can be extended as well as measurements depicting closure of opposing teeth are possible and have been proposed as occlusiometry. Thus we consider this a more effective and specific outcome from implementing digital imaging referring to odontometric techniques. In this context $3 \mathrm{D}$ images of teeth start to play an essential role in odontometric studies as the measurements are not feasible on real objects; automated digital odontometry does not exist without 3D images. That is why we pay special attention to imaging aspects. Among existing methods of morphologic description in odontology we should also mention geometric morphometrics as a method applied in various spheres for analysis of forms, shapes and sizes of biological or other objects (Richtsmeier et al., 2002), including teeth (Woods et al., 2017). The method of universality and wide coverage, having strong mathematical fundamentals depends, in terms its analytical results, largely on the input data; as if morphological features of an object are determined not by its morphology per se but rather by morphological features of group or groups. And for this reason as well we have paid special attention to interpretation, referring to dental morphology. This specific $2 \mathrm{D}$ or $3 \mathrm{D}$ data provides reasonable basis for automated orientation and landmark detection algorithms, and does not refer to measurements of other objects, besides its general approach of understanding the very basic structure.

\section{RELATED WORK}

A number of methods allow obtaining 3D images of teeth which, in their turn, can be used, in combination with automated algorithms of orientation and landmark detection, for measurements. In the variety of imaging techniques we have never applied contact methods in our studies, using primarily devices based on optical scanning (different combinations of non-contact techniques, lighting and image processing) and x-ray techniques. As the automated digital odontometry has been applied in a number of studies in dentistry and anthropology, various objects have become sources for imaging: real teeth (extracted or excavated), plaster models (separate teeth, prepared teeth, dental arches: separate or mounted on an articulator), artificially designed morphologies (physically, e.g. wax, or digitally - they do not pass through imaging stage as real objects), mandible fragments, complete mandibles and skulls. This range of objects differs by many parameters, such as optical features, size, medical ethical issues, uniqueness of finding, tooth eruption level and others. Therefore the choice of scanning device is always in strong connection with the studied object. Thus plaster arches were scanned in dental laboratory scanners; some real teeth successfully scanned in these devices as well, though others we sent to intraoral scanning directly.

However, laboratory scanners have their limitations, which have led to use of intraoral scanners. There is a variety of devices to date, among which we were interested in implementing those providing scans without spray coating. The main field of their application for automated digital odontometry, excluding dentistry of course, refers to teeth and dental arches - unique findings from anthropological (palaeoanthropological) collections. There are many pitfalls in the process of scanning teeth (Mitchell and Chadwick, 2019), especially this refers to in vivo scans. Nevertheless today a significant progress has been achieved in intraoral imaging, in terms of technological variety and scanning accuracy (Logozzo et al., 2014), (Richert et al., 2017); intraoral scanning has become an integral part of various studies, including those, oriented to measurements of teeth (Park, 2016), (Ferrini et al., 2019).

However, imaging of anthropological material is free from some complicating factors, current in dentistry (movements of patient, presence of soft tissues or continuous salivation), optical features of enamel still remain challenging. Application of intraoral cameras has prompted a number of studies where properties of images are assessed as well. Thus resolution is associated with number of triangles per area on a digital model (Solaberrieta et al., 2016) or a minimal distance between two points to be differentiated (Maret et al., 2014). Accuracy is referred as combination of deviation and resolution (Solaberrieta et al., 2016). A number of studies carried out to estimate intraoral scanners' accuracy have come to the conclusion that it depends on the size of the object; thus single tooth scans or partial arch scans (up to four units) have excellent and acceptable accuracy. However, full arch scans inevitably give distortions, calculated, for instance, by combining images in bestfit alignment (Solaberrieta et al., 2016), (Abduo and Elseyoufi, 2018). There is a variety of opinions caused by implementing of different approaches for accuracy assessment, which, in its turn, depends largely on clinical or other applications being discussed in studies. Thus not only digital images but 3D printed models become acceptable for accuracy assessments, measurements carried out by digital calipers and comparisons (Brown et al., 2018). Similar studies, which have been carried out on dried mandibles are of particular interest due to their similarity to anthropological material (Jacob et al., 2015).

Insofar as optical scanning is limited to visible parts of the studied objects, but areas of interest are beyond them, a serious part 3D imaging in our studies is based on $\mathrm{x}$-ray vision and image processing. Thus Cone Beam Computed Tomography (CBCT) is in period of its rapid development, especially in dental practice. Its principles derive from early 60s-70s (Hounsfield, 1973) and continue in palaeoanthropological studies as well (Uldin, 2017). It should be mentioned that cone beam computed tomography produces high quality 3D images of bone structures without high exposure of patients to x-rays. Of course, radiation dose is not the most significant factor in palaeoanthropological studies unless this refers to sensitive organic substances (thus DNA analyses should be performed preferably before scanning, especially on non-CBCT tomography scanners); image quality and appropriate software play a more significant part. In CBCT scanning image quality is a combination of factors including exposure protocols, radiation dose ranges, spatial resolution (voxel size), segmentation accuracy and Hounsfield unit standardization. However, a $200 \mu \mathrm{m}$ accuracy level is considered an acceptable result for CBCT scans (Maret et al., 2014); intraoral scanners provide significantly higher accuracy (Jacobs et al., 2018), which make them preferable for scanning such small objects as coronal parts of teeth (including their morphological structures). Other studies, referring predominantly to orthodontics, analyze measurements on dental arches (usually stone cast models poured from alginate impressions are compared to CBCT-obtained images), considering differences even more than $0.2 \mathrm{~mm}$ at large field of 
view (FoV) can be considered as clinically acceptable threshold in orthodontic practice (Maroua et al., 2016), which means that researchers or clinicians intensions have a strong influence on data interpreting. This is supported by other studies which suggest measurements, based on skeletal (or soft tissue) landmarks require critical evaluation (Lisboa et al., 2015). CBCT obtained image processing allows segmentation of teeth out of 3D reconstruction, which is performed in semi-automated mode (Sang et al., 2016). Though such images provide essential information on root morphology (which is planned to be studied by automated digital odontometry in the near future), significant deviations in linear parameters are reported if compared to the same teeth (extracted later for orthodontic directions) measured by calipers and on images, obtained by intraoral scanner. Such deviations are explained by location of areas of expressed CBCT image distortions on root apex and grooves on occlusal surface (due to low contrast to noise ratio). For that very reason we find images, obtained from x-ray micro tomography scanners more suitable for automated digital odontometry. Just as accuracy of different CBCT scans can be assessed in association of voxel size, field of view (FoV) and topographic localization of image discrepancies if compared to high precision x-ray micro tomography (microCT) reference images (Maret et al., 2014). Micro CT images are not only capable of reflecting surface morphological details of teeth, but also provide data for measurements of dentine structures.

\section{METHODS}

Various scanning methods have been implemented during automated digital odontometric studies carried out in dentistry and anthropology (palaeoanthropology). Imaging of teeth: their coronal parts, where this was the only available data (plaster models or in vivo optical scanning), or their complete, including roots, structure, has been necessary for providing material for the mentioned studies. Plaster models at early stages of method development were scanned on photogrammetric system including structured light designed at GosNIIAS (Knyaz and Zheltov, 2008, Knyaz, 2012); currently it is used for scanning skulls (Knyaz et al., 2019). Plaster models were scanned on dental laboratory scanners as well (Evolution plus, Zfx; S600 Arti Scanner, ZirkonZahn), which provide high degree of image accuracy. In cases when application of scan spray was not restricted and teeth were fixed in alveolae, anthropological samples of appropriate size were subjected to scanning in such scanners (Figure 1 ).

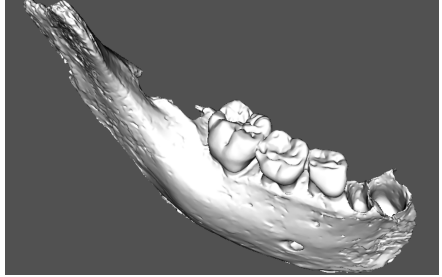

(a) mandible fragment

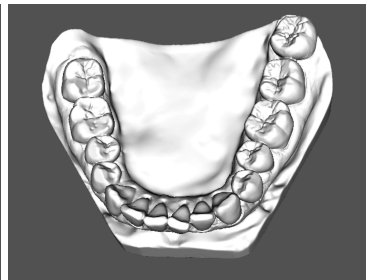

(b) plaster model of lower dental arch
Figure 1. Mandible fragment (modern history) and plaster model of lower dental arch images: laboratory scanners

For other applications in anthropological studies as well as in dentistry intraoral scanners were used. Their principal advantages for our studies are high accuracy in single tooth of limited arch area scanning, accompanied by ability of enamel scanning without opaque spray application. These features are combined in Trios (3Shape) intraoral scanner; construction is based on confocal optics. The scanner was used mainly for imaging teeth on unique findings for palaeoanthropological applications (Figure 2).

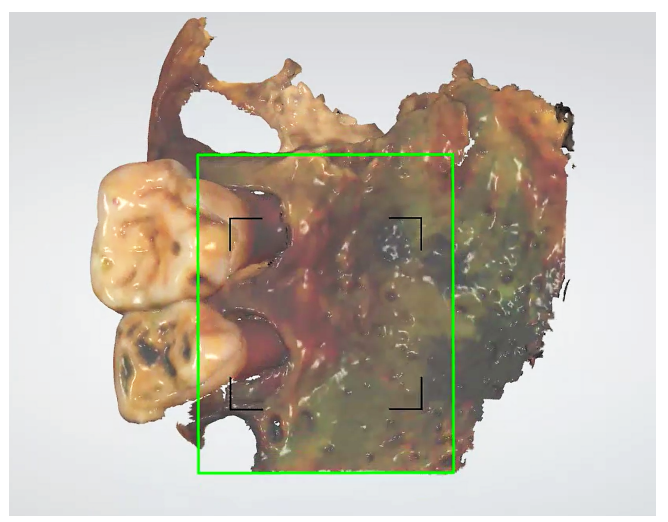

Figure 2. Intraoral camera scanning Neolithic maxilla fragment with two deciduous teeth

Obvious limitations of optical scanning methods and hidden structures related to studied objects - teeth have led to a series of imaging tests on CBCT and micro-CT scanners. Providing valuable information on bone structures and root morphology (Figure 3), CBCT scans (Vatech PaX-i 3D; 0.2x0.2x0.2; 550x550x450) were used for segmentation of tooth from the scan, which could not had been removed from the mandible fragment for alternative scanning without sample damaging. This came to light after several gentle attempts of removing the tooth from its socket.

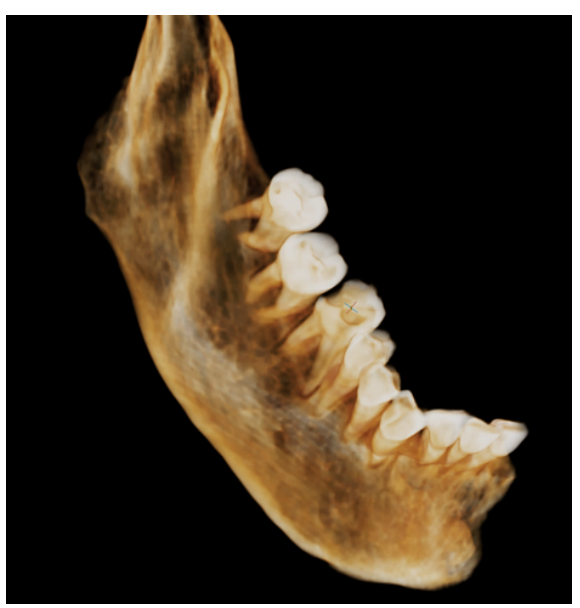

Figure 3. Mandible fragment CBCT scan (Early Bronze)

Tooth segmentation was performed by Ravil M. Galeev (Institute of Ethnology and Anthropology of Russian Academy of Sciences) on mandible fragment (Early Bronze Age). Segmentation performed on Inobitec DICOM (Russian Federation) software allowed to extract image of molar (4.7) from the scan. As the mandible fragment had been previously scanned by intraoral camera, we had an opportunity to compare, if not the whole images of the tooth, but its coronal part. Preliminary image combining was performed for accuracy assessment, but not continued due to obvious results showing discrepancy (Figure 4). Intraoral scanner should be used for surface scanning rather than CBCT. 


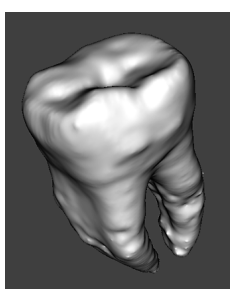

(a) segmented from (b) cut from intraoral CBCT

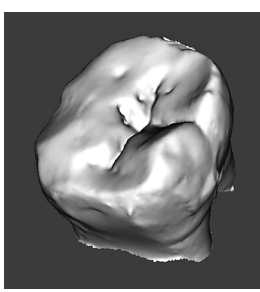

scan

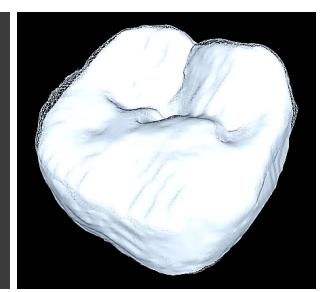

(c) combined both
Figure 4. Segmented from CBCT, cut from intraoral scan, combined both, 4.7 molar (Early Bronze)

However, in terms of scanning accuracy, there are methods of optical and x-ray scanning which can be compared and applied on equal terms for various measurements (coronal part, root, dentine basis) by means of automated digital odontometry. This refers to $\mathrm{x}$-ray micro tomography scans, which provide very detailed and accurate information, especially when teeth can be easily (due to their position and morphology) removed from the studied object. Referring to the previously described mandible fragment, premolar (4.4) was scanned by both, intraoral and micro-CT, scanners (Figure 5).

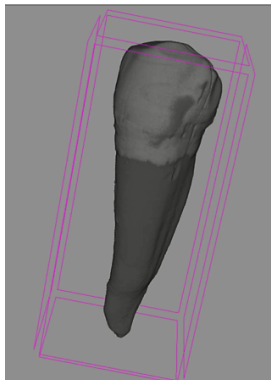

(a) micro-CT

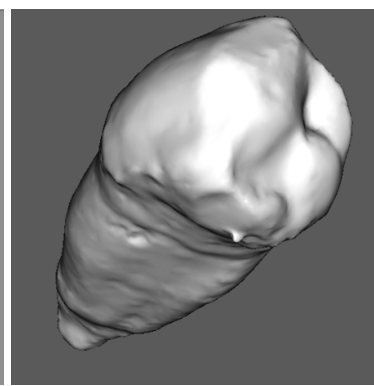

(b) intraoral
Figure 5. Micro-CT and intraoral scans of 4.4 premolar (Early Bronze)

\section{RESULTS AND DISCUSSION}

Dealing with various objects subjected to 3D imaging for further odontometric studies and variety in study cases (dental or anthropological), we can say that every technique has its pros and cons. Involvement of plaster models of teeth and dental arches in digital odontometric studies took place for medical ethical reasons in experimental studies of tooth preparation. Custom designed photogrammetric system, including structured lighting, and dental laboratory scanners were used for imaging purposes. Such devices provide accurate models due to stability (camera or table, depending on device) and calibration procedure. Therefore we preferred such devices for scanning teeth, but due to tooth enamel translucency and lighting features this was possible only after covering teeth by opaque spray, thereby scanning of unique anthropological material in such devices was excluded. Another limitation of laboratory scanners is in object size; designed for scanning of plaster models of dental arches they can cope with mandible fragment, sometimes even mandibles, but definitely not skulls, containing dental arches and teeth as subject of studies. As we mentioned, some scanners' construction includes rotating tables. This is a potential source of image distortions while scanning dry material due to lack of ligaments surrounding roots and fixing them to bone tissue. We should mention that in a number of studies, due to stability and accuracy of laboratory scanning, images obtained through them serve as reference images for comparison with intraoral scanning; though laboratory scanning and plaster model fabrication can themselves serve as material for studies of accuracy. With regard to comparisons of extraoral and intraoral scanner accuracies, based on measurements performed by experts on real objects and their 3D images, there are studies which are not in favour of fixed scanner. Such an unusual, from expectations point of view, result might be caused, if we take into consideration our experience of scanning, by two factors: mobility of rotating table of the fixed scanner and absence of periodontal ligaments on dried anthropological material, which, in their turn, can cause registered distortions. Referring to intraoral scanning, it has become the best means for imaging teeth with their enamel translucency, either in vivo, or cases related to anthropology. Intraoral scanners are not suited for large objects (e.g., skull-size object cannot be scanned by intraoral camera), and, as studies show, image distortion grows proportionally to scanned area. However separate teeth or limited areas within arches can be scanned accurately, which, for now at least, matches with study objectives of automated digital odontometry, focused on coronal parts of teeth. It should be mentioned that intraoral scanners have a high potential for application in anthropological field research as well. Though such applications require training procedure, as our experience has showed that imaging process and result depend not only on technical feature of the manually operated scanner but the operator skills as well. Images of teeth obtained or segmented from CBCT scans apparently possess sufficient accuracy for orthodontic studies. They also possess inaccessible through other methods (if we take into consideration in vivo studies) data on root morphology, which we are planning to make a part of further odontometric studies. CBCT, or even medical computed tomography, remain leaders in scanning objects in their segment due to two essential features: low dose and time of examination. However, images of tooth crowns studied by automated digital odontometry, have appropriate requirements in reflecting morphological features, and here CBCT images cannot be considered detailed in comparison to optical scanning and x-ray micro tomography; their accuracy is order of magnitude higher and thus provides for correct measurement results.

\section{CONCLUSION}

Imaging method selection is always in strong connection with multiple features of the studied material, as well as the study objectives. As the method of automated digital method provides for odontometric studies a range of objective and precise data, thick enough to describe dental morphology through measurements, we are interested in obtaining accurate and detailed images of the studied object, as they determine the accuracy of measurements. Thus according to our latest scanning tests of studied material, there is a strong tendency for application intraoral and micro-CT imaging in odontometric studies. At the same time accurate information can be obtained from laboratory and CBCT scanners for plaster and in vivo imaging tasks respectively.

\section{ACKNOWLEDGEMENTS}

The work was performed with the support by Grant 17-29-04509 of Russian Foundation for Basic Research (RFBR). 


\section{REFERENCES}

Abduo, J. and Elseyoufi, M., 2018. Accuracy of intraoral scanners: A systematic review of influencing factors. The European journal of prosthodontics and restorative dentistry.

Brown, G., Currier, G., Kadioglu, O. and Kierl, J., 2018. Accuracy of 3-dimensional printed dental models reconstructed from digital intraoral impressions. American Journal of Orthodontics and Dentofacial Orthopedics 154, pp. 733-739.

Ferrini, F., Sannino, G., Chiola, C., Capparè, P., Gastaldi, G. and Gherlone, E., 2019. Influence of intra-oral scanner (i.o.s.) on the marginal accuracy of cad/cam single crowns. International Journal of Environmental Research and Public Health 16, pp. 544.

Gaboutchian, A. V., Knyaz, V. A., Leybova, N. A., Petrosyan, G., Simonyan, H. and Vasilyev, S. V., 2019. Application of photogrammetric techniques in palaeoodontological studies through automated digital shape analysis of human teeth. ISPRS - International Archives of the Photogrammetry, Remote Sensing and Spatial Information Sciences XLII-2/W12, pp. 75-80.

Hounsfield, G. N., 1973. Computerized transverse axial scanning (tomography): Part 1. description of system. The British Journal of Radiology 46(552), pp. 1016-1022. PMID: 4757352.

Irish, J. D. and Scott, G., 2015. A Companion to Dental Anthropology.

Jacob, H., Wyatt, G. and Buschang, P., 2015. Reliability and validity of intraoral and extraoral scanners. Progress in Orthodontics.

Jacobs, R., Salmon, B., Codari, M., Hassan, B. and Bornstein, M., 2018. Cone beam computed tomography in implant dentistry: Recommendations for clinical use. BMC Oral Health.

Knyaz, V. A., 2012. Image-based 3d reconstruction and analysis for orthodontia. ISPRS - International Archives of the Photogrammetry, Remote Sensing and Spatial Information Sciences XXXIX-B3, pp. 585-589.

Knyaz, V. A. and Zheltov, S., 2008. Photogrammetric techniques for dentistry analysis, planning and visualisation. ISPRS - International Archives of the Photogrammetry, Remote Sensing and Spatial Information Sciences XXXVII-B5, pp. 783-788.

Knyaz, V. A., Maksimov, A. A. and Novikov, M. M., 2019. Vision based automated anthropological measurements and analysis. ISPRS - International Archives of the Photogrammetry, Remote Sensing and Spatial Information Sciences XLII-2/W12, pp. 117-122.

Lisboa, C. d. O., Masterson, D., Motta, A. F. J. and Motta, A. T., 2015. Reliability and reproducibility of three-dimensional cephalometric landmarks using cbct: a systematic review. Journal of Applied Oral Science 23, pp. 112-119.

Logozzo, S., Zanetti, E., Franceschini, G., Kilpela, A. and Mäkynen, A., 2014. Recent advances in dental optics. part i: $3 \mathrm{~d}$ intraoral scanners for restorative dentistry. Optics and Lasers in Engineering 54, pp. 203-221.

Maret, D., Peters, O., Galibourg, A., Dumoncel, J., Esclassan, R., Kahn, J.-L., Sixou, M. and No, T., 2014. Comparison of the accuracy of 3-dimensional cone-beam computed tomography and micro-computed tomography reconstructions by using different voxel sizes. Journal of Endodontics.

Maroua, A., Ajaj, M. and Hajeer, M. Y., 2016. The accuracy and reproducibility of linear measurements made on cbct-derived digital models. The Journal of Contemporary Dental Practice 17, pp. 294-299.
Mitchell, H. and Chadwick, R., 2019. Challenges of photogrammetric intra-oral tooth measurement. The International Archives of the Photogrammetry, Remote Sensing and Spatial Information Sciences XXXVII, part B5, pp. 779-782.

Naidu, D. and Freer, T., 2013a. The evidence supporting methods of tooth width measurement: Part i. vernier calipers to stereophotogrammetry. Australian orthodontic journal 29, pp. 159-63.

Naidu, D. and Freer, T., 2013b. The evidence supporting methods of tooth width measurement: Part ii. digital models and intra-oral scanners. Australian orthodontic journal 29, pp. 164-9.

Park, J.-M., 2016. Comparative analysis on reproducibility among 5 intraoral scanners: Sectional analysis according to restoration type and preparation outline form. The Journal of Advanced Prosthodontics 8, pp. 354-362.

Rajshekar, M., Julian, R., Williams, A.-M., Tennant, M., Forrest, A., Walsh, L., Wilson, G. and Blizzard, L., 2017. The reliability and validity of measurements of human dental casts made by an intra-oral 3d scanner, with conventional hand-held digital callipers as the comparison measure. Forensic Science International.

Richert, R., Goujat, A., Venet, L., Viguie, G., Viennot, S., Robinson, P., Farges, J.-C., Fages, M. and Ducret, M., 2017. Intraoral scanner technologies: A review to make a successful impression. Journal of Healthcare Engineering 2017, pp. 1-9.

Richtsmeier, J. T., Deleon, V. B. and Lele, S. R., 2002. The promise of geometric morphometrics. American Journal of Physical Anthropology 119(35), pp. 63-91.

Sang, Y.-H., Hu, H.-C., Lu, S.-H., Wu, Y., Li, W.-R. and Tang, Z.-H., 2016. Accuracy assessment of three-dimensional surface reconstructions of in vivo teeth from cone-beam computed tomography. Chinese Medical Journal 129, pp. 1464.

Shaweesh, A., 2016. Mesiodistal and faciolingual diameters of the permanent teeth in a jordanian population. Archives of Oral Biology.

Shireen, A. and Ara, S., 2016. Odontometric analysis of permanent maxillary first molar in gender determination. Journal of Forensic Dental Sciences 8, pp. 145.

Smith, R., Zaitoun, H., Coxon, T., Karmo, M., Kaur, G., Townsend, G., Harris, E. and Brook, A., 2009. Defining new dental phenotypes using 3-d image analysis to enhance discrimination and insights into biological processes. Archives of oral biology 54 Suppl 1, pp. S118-25.

Solaberrieta, E., Garmendia, A., Brizuela, A., Otegi, J. R., Pradies, G. and Szentpetery, A., 2016. Intraoral digital impressions for virtual occlusal records:section quantity and dimensions. BioMed Research International -, pp. 7.

Song, J.-W., Leesungbok, R., Park, S.-J., Chang, S., Ahn, S.J. and Lee, S.-W., 2017. Analysis of crown size and morphology, and gingival shape in the maxillary anterior dentition in korean young adults. The Journal of Advanced Prosthodontics 9, pp. 315.

Uldin, T., 2017. Virtual anthropology - a brief review of the literature and history of computed tomography. Forensic Sciences Research 2(4), pp. 165-173.

Woods, C., Fernée, C., Browne, M., Zakrzewski, S. and Dickinson, A., 2017. The potential of statistical shape modelling for geometric morphometric analysis of human teeth in archaeological research. PLOS ONE.

Zubov, A. A., 1968. Odontologiya. Metodika antropologicheskikh issledovaniy. Nauka, Moscow, p. 200. 